\title{
A Stieltjes Transform Approach for Studying the Steady-State Behavior of Random Lyapunov and Riccati Recursions
}

\author{
Ali Vakili and Babak Hassibi
}

\begin{abstract}
In this paper we study the asymptotic eigenvalue distribution of certain random Lyapunov and Riccati recursions that arise in signal processing and control. The analysis of such recursions has remained elusive when the system and/or covariance matrices are random. Here we use transform techniques (such as the Stieltjes transform and free probability) that have gained popularity in the study of large random matrices. While we have not yet developed a full theory, we do obtain explicit formula for the asymptotic eigendistribution of certain classes of Lyapunov and Riccati recursions, which well match simulation results. Generalizing the results to arbitrary classes of such recursions is currently under investigation.
\end{abstract}

\section{INTRODUCTION}

The classical time-invariant Lyapunov

$$
P_{i+1}=F P_{i} F^{*}+Q, \quad P_{0}
$$

and Riccati

$P_{i+1}=F P_{i} F^{*}-F P_{i} H^{*}\left(R+H P_{i} H^{*}\right)^{-1} H P_{i} F^{*}+Q, \quad P_{0}$

recursions arise in many applications, and their convergence properties are well understood. However, their time varying counterparts (with the matrices $F, H, Q, R$ all replaced by time-varying $F_{i}, H_{i}, Q_{i}, R_{i}$ ) are much more difficult to analyze and depend on the nature of the time-variation of the system and covariance matrices.

An important class of such time-varying recursions arise when the matrices $F_{i}, H_{i}, Q_{i}, R_{i}$ are drawn from some random matrix ensemble. In this case, the $P_{i}$ will form a matrix-valued random process. When the $F_{i}, H_{i}, Q_{i}, R_{i}$ are themselves drawn from jointly stationary matrix-valued random processes, one may suspect that the $P_{i}$ will also be asymptotically stationary, i.e., the process $\left\{P_{i}\right\}$ will converge to a stationary process in the limit as $i \rightarrow \infty$. However, demonstrating this and, more importantly, determining the stationary distribution of $P_{i}$ appears to be quite challenging.

Examples of where such random recursions arise include adaptive filtering where the regressors are often drawn randomly [1], [2], [3] and control over packet-dropping links (where the covariance matrices $R_{i}$ and $Q_{i}$ are chosen randomly according to whether packets are dropped or not) [4],

This work was supported in part by the National Science Foundation under grant CCF-0729203, by the David and Lucille Packard Foundation, and by Caltech's Lee Center for Advanced Networking.

Ali Vakili is with Electrical Engineering Department, California Institute of Technology, 1200 E California Blvd., Pasadena, CA 91125, USA avakili@caltech.edu

Babak Hassibi is with Faculty of Electrical Engineering Department, California Institute of Technology, 1200 E California Blvd., Pasadena, CA 91125, USA hassibiecaltech.edu
[5]. However, determining the asymptotic behavior of these recursions can be quite challenging (e.g., in [4] determining the mean of $P_{i}$-in fact, even determining the stability of $P_{i}$-remains open).

While determining the entire asymptotic distribution of $P_{i}$ may be well beyond reach, determining other aspects of $P_{i}$ may be tractable. In this paper, we propose to use ideas from the theory of large random matrices (especially, transform theory) to say things about the asymptotic distribution of the eigenvalues of $P_{i}$ in various random Lyapunov and Riccati recursions. As is the case in large random matrix theory, the theory requires that the size of the underlying matrices be large. While we have not yet been able to develop a full theory for this-something that in any event may be too ambitious, - we are able to obtain non-trivial new results for certain classes of Lyapunov and Riccati recursions. These also well match results from simulations. We believe that the results we have obtained here show the promise of large random matrix theory techniques in studying random matrix recursions and are therefore worthy of further scrutiny.

The remainder of this paper is organized as follows. In Section II we review a number of results from random matrix theory which appear to be useful in our study. In Section III we will look at random Lyapunov recursions and will obtain the steady-state eigendistribution for three important forms of these recursions. In Section IV a number of random Riccati recursions which arise in different problems are investigated. Finally, Section V concludes the paper.

\section{DEFINITIONS AND NotATION}

In this section we will present a few definitions and notational conventions which will be used throughout this work. For an $n \times n$ random matrix $\mathrm{M}$ the empirical distribution of the eigenvalues is defined as,

$$
F_{\mathrm{M}}(x)=\frac{1}{n} \sum_{l=1}^{n} \mathbb{1}\left\{\lambda_{l}(\mathrm{M}) \leq x\right\},
$$

where $\lambda_{l}(\mathbf{M})$ is the $l$-th eigenvalue of $\mathbf{M}$ and $\mathbb{1}(\cdot)$ denotes the Heaviside step function. An empirical distribution function $f_{M}(x)$ can also be associated with $F_{\mathrm{M}}(x)$. This density function is frequently referred to as the eigendistribution of M.

Many results on the eigendistribution of random matrices are expressed in terms of some transform of $f_{M}(\lambda)$. The most important of these transforms is the so-called Stieltjes transform which is defined as,

$$
S_{\mathrm{M}}(z)=\mathbb{E}\left[\frac{1}{\lambda-z}\right] \text {. }
$$


Equivalent definitions, which are often useful, include

$$
S_{\mathrm{M}}(z)=\mathbb{E} \frac{1}{n} \operatorname{tr}(\mathrm{M}-z \mathrm{I})^{-1}
$$

and

$$
S_{\mathrm{M}}(z)=\frac{d}{d z} \mathbb{E} \frac{1}{n} \log \operatorname{det}(z \mathbf{I}-\mathbf{M})^{-1} .
$$

We will refer to $S_{\mathrm{M}}(z)$ as the Stieltjes transform of the matrix $M$ or the Stieltjes transform of its eigendistribution interchangeably. Having the Stieltjes transform, we can retrieve the eigendistribution uniquely through its inversion formula [6],

$$
f_{\mathrm{M}}(\lambda)=\lim _{\omega \rightarrow 0^{+}} \frac{1}{\pi} \operatorname{Im}\left[S_{\mathrm{M}}(\lambda+j \omega)\right] .
$$

We can also obtain the various moments of the eigendistribution of M through power series expansion of the Stieltjes transform. Expanding around infinity yields the moments $m_{i}=\mathbb{E} \lambda^{i}, i=0,1, \ldots$

$$
S_{\mathrm{M}}(z)=-\sum_{i=0}^{\infty} m_{i} z^{-i-1},
$$

while expanding around the origin yields the moments $m_{-i}=\mathbb{E} \lambda^{-i}, i=1,2, \ldots$

$$
S_{\mathrm{M}}(z)=\sum_{i=1}^{\infty} m_{-i} z^{i-1} .
$$

Another important transform in large random matrix theory is the R-transform which is directly related to the Stieltjes transform as,

$$
R_{\mathrm{M}}(z)=S_{\mathrm{M}}^{-1}(-z)-\frac{1}{z},
$$

where $S_{\mathrm{M}}^{-1}(\cdot)$ is the inverse function of $S_{\mathrm{M}}(\cdot)$. If expanded as a power series in terms of $z$, the coefficients of $R_{\mathrm{M}}(z)$ are referred to as the free cumulants [7]. A related fundamental concept in random matrix theory is the notion of free probability which was first introduced by Voiculescu [8]. Freeness replaces the independence property for random variables that are non-commutative (such as random matrices).

Definition II.1 (Free Random Variables). Two noncommutative random variables $X$ and $Y$ are called free if,

$$
\mathbb{E}\left[p_{1}(X) q_{1}(Y) p_{2}(X) q_{2}(Y) \ldots\right]=0,
$$

for finitely many polynomials $p_{i}$ and $q_{j}$ for which,

$$
\mathbb{E}\left[p_{i}(X)\right]=0=\mathbb{E}\left[q_{j}(Y)\right] .
$$

A key result is that for two free random variables $X$ and $Y, R_{X+Y}(z)=R_{X}(z)+R_{Y}(z)$ [9].

Definition II.2 (Gaussian-Like Random Matrices). Let $\mathrm{A}_{n \times m}$ have i.i.d. zero-mean unit-variance elements with bounded higher-order moments. Then we call $\mathrm{B}=\frac{1}{\sqrt{m}} \mathrm{~A}$ a Gaussian-like random matrix.

\section{RANDOM LYAPUNOV RECURSIONS}

As mentioned earlier, Lyapunov matrix recursions frequently appear in engineering and mathematical problems, especially in control and system theory. In its most general form, the Lyapunov matrix recursion for discrete and timevarying systems can be written as,

$$
\mathrm{P}_{i+1}=\mathrm{F}_{i}^{*} \mathrm{P}_{i} \mathrm{~F}_{i}+\mathrm{Q}_{i} .
$$

We are interested in finding the empirical distribution function of the eigenvalues of a large dimensional $\mathrm{P}_{i}$, as $i \rightarrow \infty$, while $\mathrm{F}_{i}$ and $\mathrm{Q}_{i}$ are random matrices themselves, with some known probability distributions.

Since approaching the problem in its general form seems quite formidable, we will begin by studying some special cases.

\section{A. Scalar and Non-random $F_{i}$}

We will assume that $\mathrm{F}=\sqrt{\alpha} \mathrm{I}$ is simply a scaled version of identity and that $\mathrm{Q}_{i}$ is generated by a Gaussian-like matrix, $\mathrm{H}_{i}$. More explicitly,

$$
\mathrm{P}_{i+1}=\alpha \mathrm{P}_{i}+\mathrm{H}_{i} \mathrm{H}_{i}^{*},
$$

where $\alpha \in(0,1)$ and the $\mathrm{H}_{i}$ are independently drawn $n \times m$ Gaussian-like matrices as defined in Definition II.2. It turns out that one can compute the moments of the eigendistribution of $\mathrm{P}_{i}$ in closed form. The first few moments of the empirical distribution function of $\mathrm{P}_{i}$ are given in the theorem below.

Theorem III.1. When $\alpha<1$, the matrices $P_{i}$ in (14) converge to a steady-state distribution. The first few moments of the empirical eigendistribution function of $P_{i}$ are given by

$$
\begin{aligned}
& m_{1}=\frac{1}{1-\alpha} \\
& m_{2}=\frac{1}{(1-\alpha)^{2}}+\frac{1 / \beta}{\left(1-\alpha^{2}\right)} \\
& m_{3}=\frac{1}{(1-\alpha)^{3}}+\frac{3 / \beta}{(1-\alpha)\left(1-\alpha^{2}\right)}+\frac{1 / \beta^{2}}{\left(1-\alpha^{3}\right)}
\end{aligned}
$$

where $\beta=m / n$. For $\alpha \geq 1$, the recursion (14) diverges.

Outline of Proof: Using Marcenko and Pastur's result [10] and the relation between the Stieltjes transforms of a random matrix and its scaled version, the Stieltjes transform of the eigenvalue distribution of $\mathrm{P}_{i}$ can be shown to satisfy

$$
S_{P_{i+1}}(z)=\frac{1}{\alpha} S_{P_{i}}\left(\frac{z}{\alpha}-\frac{\frac{\beta}{\alpha}}{\beta+S_{P_{i+1}}(z)}\right) .
$$

When $\alpha<1$, it is not too hard to show that the Stieltjes transform converges and therefore we have,

$$
S_{P}(z)=\frac{1}{\alpha} S_{P}\left(\frac{z}{\alpha}-\frac{\frac{\beta}{\alpha}}{\beta+S_{P}(z)}\right) .
$$

Expanding the Stieltjes transform as a power series around infinity (which brings in the $m_{i}$ ) and equating terms leads to the result of the theorem. 

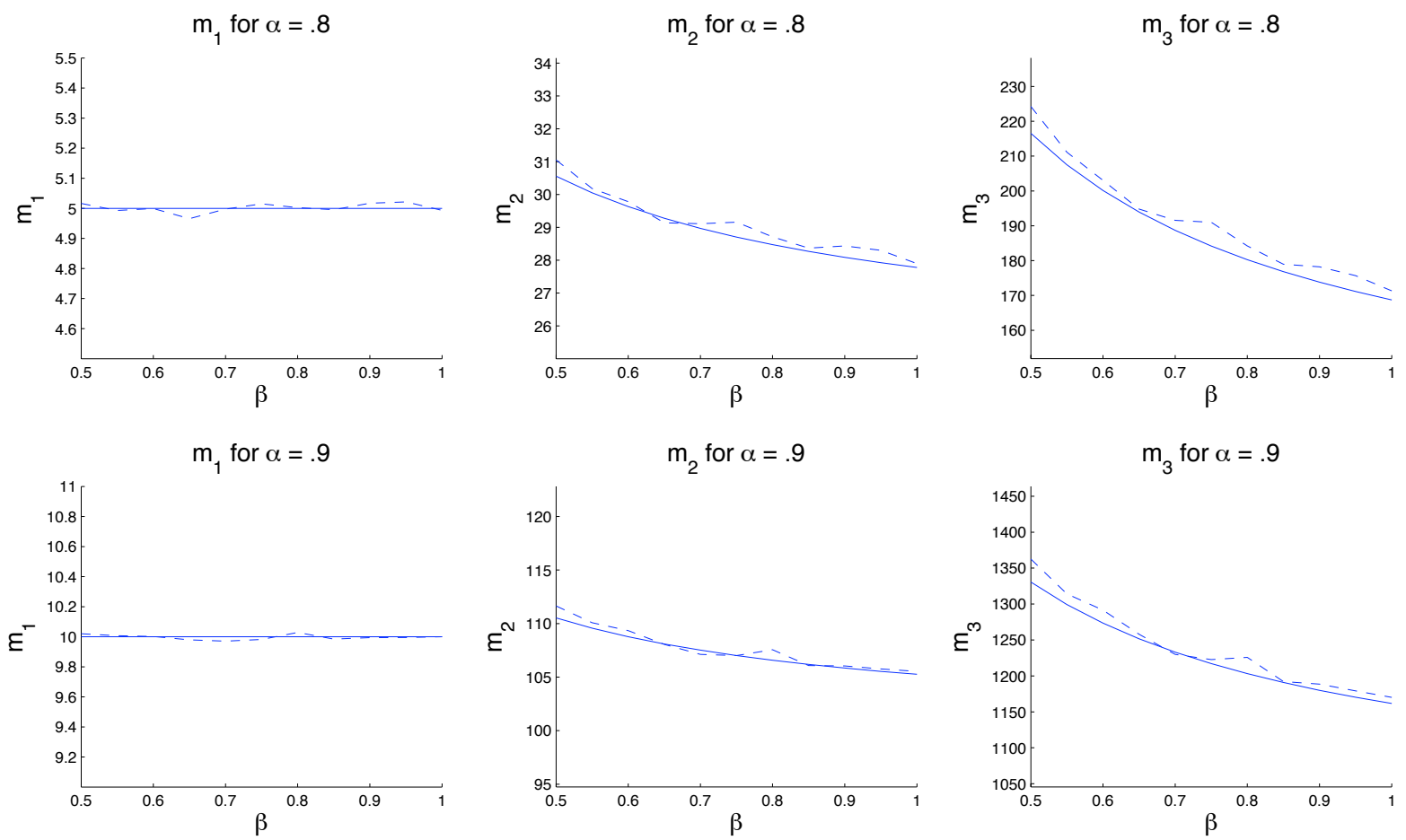

Fig. 1. Moments of the eigendistribution of $P_{i}$ in recursion (14) as a function of $\beta$ for $\alpha=0.8$ and $\alpha=0.9$ and $n=20$.

Alternatively, one can also find the first few moments using the R-transform. As mentioned in Section II, the Rtransform of the sum of two asymptotically free random variables is simply the sum of their R-transforms. Here we can readily show that given the independence of the $\mathrm{H}_{i}$ 's for different $i$ 's, $\alpha \mathrm{P}_{i}$ and $\mathrm{H}_{i} \mathrm{H}_{i}^{*}$ are free random matrices and,

$$
R_{P}(z)=\alpha R_{P}(\alpha z)+\frac{\beta}{\beta-z},
$$

where $\frac{\beta}{\beta-z}$ is the R-transform of $\mathrm{HH}^{*}$ [11]. Therefore the free cumulants of $\mathrm{P}_{i}$, or in other words the coefficients in the power series of $R_{P}(z)$ can be found to be,

$$
R_{P}(z)=\sum_{j=0}^{\infty} \frac{\beta^{-j+1}}{1-\alpha^{j}} z^{j-1}
$$

Now one can use the combinatorial relation between the free cumulants and the moments [12] to find the moments of the eigendistribution. Having the simple representation of the free cumulants, along with the systematic approach of finding the moments from them provides a relatively straightforward method for computing all the moments in this case.

Figure 1 shows a comparison of the analytic results with simulations for $\alpha=0.8$ and $\alpha=0.9$ and $n=20$. As can be seen, even though the results are for large $n$, we obtain quite good a fit for even $n=20$.

\section{B. General Lyapunov Recursions}

The above analysis for $\mathrm{F}=\sqrt{\alpha} \mathrm{I}$ can be extended to the recursions with more general $\mathrm{F}_{i}$ 's. In particular we will assume that $\mathrm{F}_{i}$ is drawn from an $n \times n$ Gaussian-like random matrix distribution (which is essentially only a condition on the first and second moments of the elements and requires the elements of $\mathrm{F}_{i}$ to be i.i.d.). Consider

$$
\mathrm{P}_{i+1}=\alpha \mathrm{F}_{i}^{*} \mathrm{P}_{i} \mathrm{~F}_{i}+\mathrm{H}_{i} \mathrm{H}_{i}^{*},
$$

in which we assume that $\mathrm{F}_{i}$ and $\mathrm{F}_{j \neq i}$ are independent. The $\mathrm{H}_{i}$ 's are assumed to be the same as in (14). Once again we can compute the moments of the eigendistribution of $\mathrm{P}_{i}$ as $i \rightarrow \infty$.

Theorem III.2. When $\alpha<1$, the eigendistribution of the matrices $P_{i}$ in (22) converges to a steady-state distribution whose Stieltjes transform satisfies the pair of implicit equations

$$
\begin{aligned}
S_{P}(z) & =\frac{1}{\alpha} t\left(\frac{z}{\alpha}-\frac{\frac{\beta}{\alpha}}{\beta+S_{P}(z)}\right) \\
t^{2}(z) & =-\frac{1}{z} S_{P}\left(-\frac{1}{t(z)}\right)
\end{aligned}
$$

The first few moments of the corresponding distribution are given by,

$$
\begin{aligned}
& m_{1}=\frac{1}{1-\alpha} \\
& m_{2}=\frac{1}{(1-\alpha)^{2}}+\frac{1 / \beta}{\left(1-\alpha^{2}\right)}+\frac{\alpha^{2}}{\left(1-\alpha^{2}\right)(1-\alpha)^{2}}
\end{aligned}
$$




$$
\begin{aligned}
m_{3} & =\frac{1}{\left(1-\alpha^{3}\right)}\left\{\left(\frac{2+m_{1}}{\beta}+\frac{1}{\beta^{2}}+1\right)+\alpha m_{1}\left(\frac{2}{\beta}+3\right)\right. \\
& \left.+\alpha^{2}\left(3 m_{1}^{2}+m_{2}\right)+\alpha^{3}\left(m_{1}^{3}+3 m_{1} m_{2}\right)\right\}
\end{aligned}
$$

where $\beta=m / n$. When $\alpha \geq 1$, the recursion (22) does not converge.

Proof: Follows the proof of Theorem III.1 by showing the convergence of the Stieltjes transform to the solution of the system of implicit equations (23) and (24). Expanding these equations as a power series in terms of $z^{-i}$ and equating the coefficients of $z^{-i}$ terms, we will obtain the moments.

\section{LMS Lyapunov Recursion}

In LMS adaptive filtering the following Lyapunov equation appears frequently [1], [2], [3],

$$
\mathrm{P}_{i+1}=\left(\mathrm{I}-\mu h_{i} h_{i}^{T}\right) \mathrm{P}_{i}\left(\mathrm{I}-\mu h_{i} h_{i}^{T}\right)+\mu^{2} r h_{i} h_{i}^{T}
$$

where the $h_{i}$ (the regressors) are independently drawn $n \times 1$ Gaussian-like vectors, $\mu$ is the so-called learning rate, and $r$ represents the variance of the measurement noise. Once again we are interested in finding the empirical eigenvalue distribution of $\mathrm{P}_{i}$, as $i \rightarrow \infty$.

In this case, it turns out that one can directly compute the Stieltjes transform of $\mathrm{P}_{i}$ (which then uniquely determines the eigendistribution according to its inversion formula (7)).

Theorem III.3. When $\mu<\frac{2}{n}$ the eigendistribution of the matrices $P_{i}$ in (28) converges to a steady-state distribution whose Stieltjes transform satisfies the differential equation,

$$
(z-\gamma) \frac{d S_{P}(z)}{d z}+S=0
$$

where,

$$
\gamma=\frac{\frac{r}{n}}{\frac{2}{n \mu}-1}
$$

This implies that $S_{P}(z)=\frac{1}{\gamma-z}$ and $p(\lambda)=\delta(\lambda-\gamma)$, i.e., as $n \rightarrow \infty P_{i}$ converges to $\gamma I$. When $\mu>\frac{2}{n}$ the recursion (28) diverges.

Outline of Proof: One can first show convergence in distribution for $\mu<\frac{2}{n}$. Once we have this convergence, we apply the definition (5) to compute the Stieltjes transform to the both sides of (28). Using the Matrix inversion lemma, which gives the inverse of a matrix $A=B+C D C^{T}$ as

$$
A^{-1}=B^{-1}-B^{-1} C\left(D^{-1}+C^{T} B^{-1} C\right)^{-1} C^{T} B^{-1},
$$

we obtain

$$
S_{P_{i+1}}(z)=S_{P_{i}}(z)-\frac{1}{n} \mathbb{E} \operatorname{tr}\left\{M_{1} M_{2}^{-1} M_{1}^{T}\right\},
$$

where

$$
M_{1}=\left(\mathrm{P}_{i}-z \mathrm{I}\right)^{-1}\left[\begin{array}{ll}
h_{i} & \mathrm{P}_{i} h_{i}
\end{array}\right]
$$

and

$$
\begin{aligned}
M_{2}= & {\left[\begin{array}{cc}
\mu^{2}\left(r+h_{i}^{T} \mathrm{P}_{i} h_{i}\right) & -\mu \\
-\mu & 0
\end{array}\right]^{-1}+} \\
& {\left[\begin{array}{c}
h_{i}^{T} \\
h_{i}^{T} \mathrm{P}_{i}
\end{array}\right]\left(\mathrm{P}_{i}-z \mathbf{I}\right)^{-1}\left[\begin{array}{ll}
h_{i} & \mathrm{P}_{i} h_{i}
\end{array}\right] . }
\end{aligned}
$$

Using the self-averaging property of large random matrices [13],

$$
\lim _{n \rightarrow \infty} h_{i}^{T}\left(\mathrm{P}_{i}-z \mathrm{I}\right)^{-1} h_{i}=S_{P_{i}}(z) \quad \text { a.s., }
$$

and its direct consequence,

$$
\lim _{n \rightarrow \infty} h_{i}^{T}\left(\mathrm{P}_{i}-z \mathrm{I}\right)^{-2} h_{i}=\frac{d S_{P_{i}}(z)}{d z} \quad \text { a.s. }
$$

we can rewrite (32) in terms of the Stieltjes transform and its derivative. With some computational effort, the differential equation (29) will be obtained for the Stieltjes transform of $\mathrm{P}_{i}$ as $i \rightarrow \infty$. For $\mu \geq \frac{2}{n}$, divergence follows from (30), since $\gamma$ becomes negative.

As can be seen from the simulations in Figure 2, this theorem correctly predicts the accumulation of all the eigenvalues of $\mathrm{P}_{i}$ at a single point. This is also consistent with what is expected intuitively.

\section{RANDOM RICCATI RECURSIONS}

Riccati recursions are found in various forms in systems and control theory, estimation theory and many other fields. When $\mathrm{P}_{i}$ is invertible the Riccati recursion can be written as,

$$
\mathrm{P}_{i+1}=\mathrm{F}_{i}^{*}\left(\mathrm{P}_{i}^{-1}+\mathrm{H}_{i} \mathrm{H}_{i}^{*}\right)^{-1} \mathrm{~F}_{i}+\mathrm{Q}_{i} .
$$

As in the Lyapunov case, we are interested in determining the asymptotic eigenvalue structure of $\mathrm{P}_{i}$ for large dimensional matrices. Once again, the general case appears to be quite challenging and we begin by considering special cases, a number of which are given below.

\section{A. A simple Riccati Recursion with $F=I, Q=I$.}

In the case of $\mathrm{F}_{i}=\mathrm{I}$ and $\mathrm{Q}_{i}=\mathrm{I}$, (38) simplifies to

$$
\mathrm{P}_{i+1}=\left(\mathrm{P}_{i}^{-1}+\mathrm{H}_{i} \mathrm{H}_{i}^{*}\right)^{-1}+\mathrm{I} \text {. }
$$

Assume that the $\mathrm{H}_{i}$ are independently drawn Gaussian-like $n \times m$ matrices with $\beta=\frac{m}{n}$. In this case, the Stieltjes transform of the limiting eigenvalue distribution can be found from a system of implicit equations.

Theorem IV.1. As $i \rightarrow \infty$, the eigendistribution of $P_{i}$ in (39) converges to a stationary distribution whose Stieltjes transform satisfies the following system of implicit equations,

$$
\begin{aligned}
& v(z)=-\frac{1}{z}-\frac{1}{z^{2}} S_{P}\left(z^{-1}\right) \\
& u(z)=v\left(z-\frac{\beta}{\beta+u(z)}\right) \\
& S_{P}(z+1)=-\frac{1}{z}-\frac{1}{z^{2}} u\left(z^{-1}\right)
\end{aligned}
$$



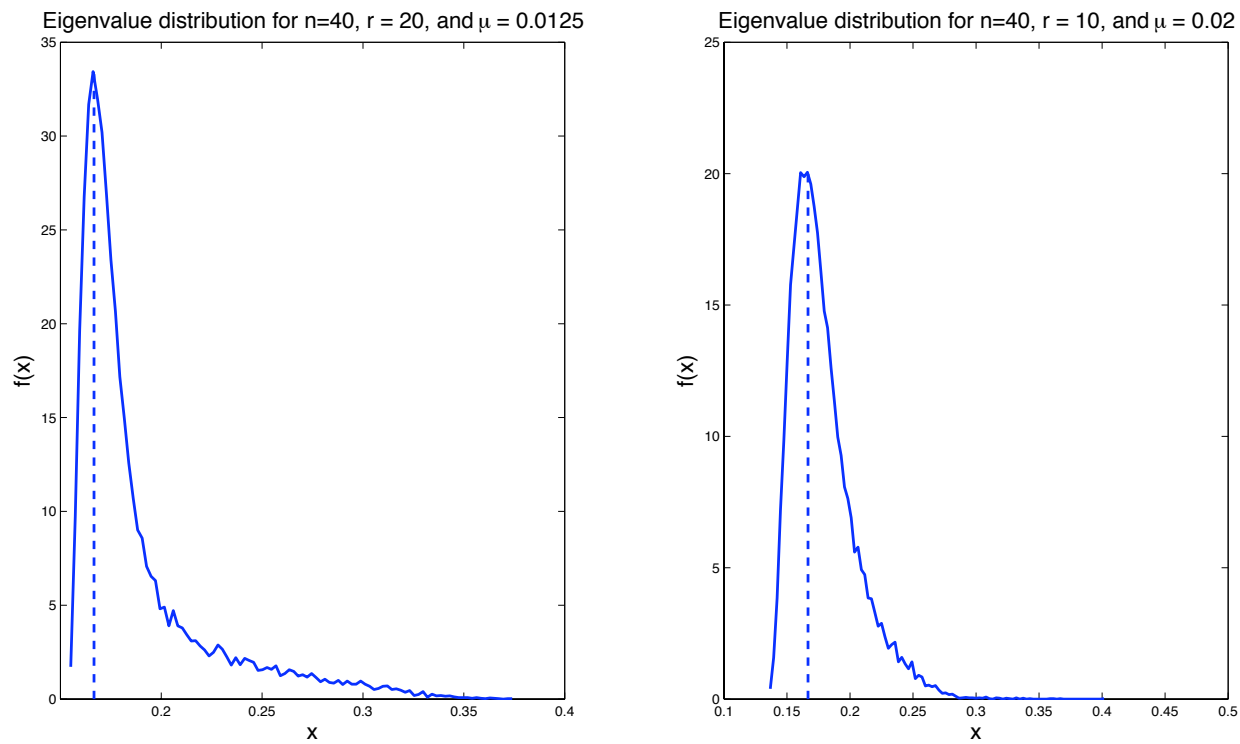

Fig. 2. The eigendistribution of $P_{i}$ in the Lyapunov recursion (28).

Proof: The proof uses Marcenko and Pastur's result [10] along with the relation between the Stieltjes transforms of eigendistributions of a matrix and its inverse, namely,

$$
S_{P^{-1}}(z)=-\frac{1}{z}-\frac{1}{z^{2}} S_{P}\left(z^{-1}\right) .
$$

Attempting to solve explicitly the system of implicit equations in Theorem IV.1 seems difficult. How to best find $S_{P}(z)$ numerically is currently under investigation.

Following the above approach, we can also find the eigendistribution for a more general Riccati recursion,

$$
\mathrm{P}_{i+1}=\alpha \mathrm{F}_{i}^{*}\left(\mathrm{P}_{i}^{-1}+\mathrm{H}_{i} \mathrm{H}_{i}^{*}\right)^{-1} \mathrm{~F}_{i}+\mathrm{I},
$$

where $\mathrm{F}_{i}$ 's are $n \times n$ Gaussian-like random matrices. Each $\mathrm{F}_{i}$ is independent of all $\mathrm{F}_{j \neq i}, \alpha$ is a constant scalar, and $\mathrm{H}_{i}$ are as in (39).

Theorem IV.2. As $i \rightarrow \infty$, the eigendistribution of $P_{i}$ in the Riccati recursion of (44) converges to a stationary distribution whose Stieltjes transform satisfies the following system of implicit equations

$$
\begin{aligned}
v(z) & =-\frac{1}{z}-\frac{1}{z^{2}} S_{P}\left(z^{-1}\right) \\
u(z) & =v\left(z-\frac{\beta}{\beta+u(z)}\right) \\
w(z) & =-\frac{1}{z}-\frac{1}{z^{2}} u\left(z^{-1}\right) \\
\alpha^{2} z S_{P}^{2}(\alpha z+1) & =-w\left(\frac{-1}{\alpha S_{P}(\alpha z+1)}\right)
\end{aligned}
$$

\section{B. A Riccati Recursion with Low-Rank Update}

As another interesting special case of the Riccati recursion, we look at the eigendistribution of $\mathrm{P}_{i}$ which satisfy the following recursion

$$
\mathrm{P}_{i+1}=\left(\mathrm{P}_{i}^{-1}+\frac{1}{r} h_{i} h_{i}^{*}\right)^{-1}+q g_{i} g_{i}^{*}
$$

where the $h_{i}$ and $g_{i}$ are independently drawn $n \times 1$ Gaussianlike vectors and $r$ and $q$ are constant parameters (This Riccati recursion arises, for example, in $H^{\infty}$ adaptive filtering [14]).

In this case, we can directly obtain an expression for the Stieltjes transform of $\mathrm{P}_{i}$.

Theorem IV.3. As $i \rightarrow \infty$, the eigendistribution of $P_{i}$ in (49) converges to a stationary distribution whose Stieltjes transform satisfies the equation

$$
z^{2} S(z)-\left(q^{-1} z^{2}+z-r\right) S(z)+q^{-1} z+c=0,
$$

where $c$ is a constant.

Outline of Proof: Convergence of the eigendistribution of $\mathrm{P}_{i}$ as $i \rightarrow \infty$ can be shown. When this is the case, we can proceed to find the Stieltjes transform of the steady-state eigendistribution. To this end, using matrix inversion lemma we can rewrite (49) as

$$
\mathrm{P}_{i+1}=\mathrm{P}_{i}-\frac{\mathrm{P}_{i} h_{i} h_{i}^{*} \mathrm{P}_{i}}{r+h_{i}^{*} \mathrm{P}_{i} h_{i}}+q g_{i} g_{i}^{*}
$$




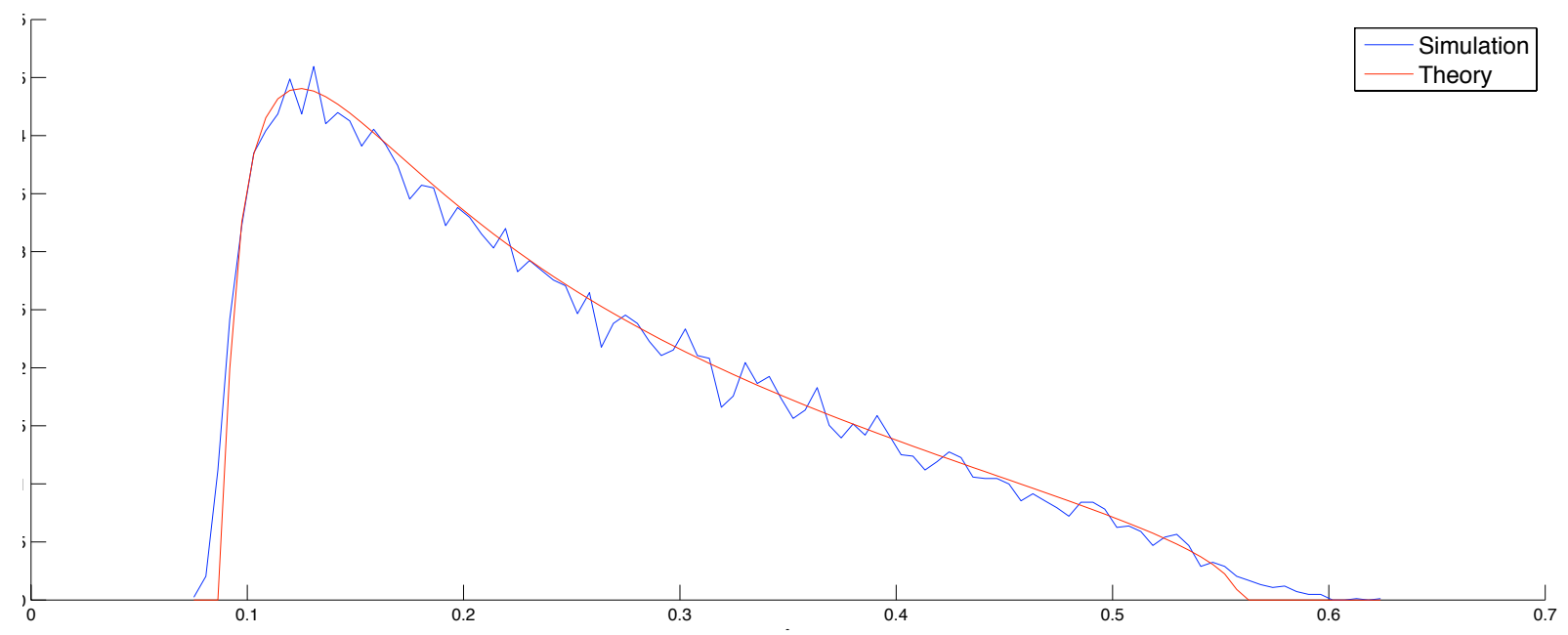

Fig. 3. The steady-state eigendistribution of the recursion (49) for $n=20$ and $r=0.5$.

Then we will employ the same techniques used in the proof of Theorem III.3. $M_{1}$ and $M_{2}$ in (32) will be replaced by the matrices

$$
M_{1}^{\prime}=\left(\mathrm{P}_{i}-z \mathrm{I}\right)^{-1}\left[\begin{array}{ll}
g_{i} & \mathrm{P}_{i} h_{i}
\end{array}\right]
$$

and

$$
\begin{aligned}
M_{2}^{\prime}= & {\left[\begin{array}{cc}
q & 0 \\
0 & -\frac{1}{r+h_{i}^{*} \mathrm{P}_{i} h_{i}}
\end{array}\right]^{-1}+} \\
& {\left[\begin{array}{c}
g_{i}^{*} \\
h_{i}^{*} \mathrm{P}_{i}
\end{array}\right]\left(\mathrm{P}_{i}-z \mathrm{I}\right)^{-1}\left[\begin{array}{ll}
g_{i} & \mathrm{P}_{i} h_{i}
\end{array}\right] }
\end{aligned}
$$

Now one can rewrite the counterpart of (32) in terms of the Stieltjes transform and its derivative (once again, using the self-averaging properties,) and (50) will be obtained after some calculations.

If we attempt to find $c$ using the series expansion of $S(z)$, we obtain

$$
c=\frac{m_{1}}{q}
$$

Thus $c$ cannot be separately determined. The reason is that the Riccati recursion is nonlinear and there is no simple algebraic equation for $m_{1}=\frac{1}{n} \mathbb{E} \operatorname{tr} \mathrm{P}_{i}$. Solving the quadratic equation of (50) for $S(z)$, and using the inverse formula for the Stieltjes transform yields

$$
p_{\lambda}(\lambda)=\frac{1}{\pi} \operatorname{Im}\left(\frac{\sqrt{\left(q^{-1} \lambda^{2}+\lambda-r\right)^{2}-4 \lambda^{2}\left(q^{-1} \lambda+c\right)}}{2 \lambda^{2}}\right) .
$$

The constant $c$ is then found such that the resulting $p_{\lambda}(\cdot)$ is a valid distribution, i.e., $\int p(\lambda) d \lambda=1$. Fig. 3 shows the eigendistribution obtained from simulations for $n=20$ and $r=0.5$. We have obtained the theoretical curve by assuming $S(z)=u+j v$ and numerically solving for $u$ and $v$ while forcing the distribution to integrate to one.

\section{CONCLUSIONS}

In this paper, we employed techniques from the theory of large random matrices to obtain various results on the asymptotic eigendistribution of certain classes of random Lyapunov and Riccati recursions. The preliminary results seem encouraging and suggest that they are worthy of further scrutiny and pursuit.

\section{REFERENCES}

[1] S. Haykin, Adaptive Filter Theory, Prentice-Hall, 2001.

[2] A.H. Sayed, Fundamentals of Adaptive Filtering, John Wiley \& Sons, 2003.

[3] T. Kailath, A.H. Sayed, and B. Hassibi, Linear Estimation, PrenticeHall, 2000.

[4] B. Sinopoli, L. Schenato, M. Franceschetti, K. Pooll, M. Jordan, and S. Sastry, "Kalman filtering with intermittent observations," IEEE Transactions on Automatic Control, vol. 49, no. 9, pp. 1453-1464, Sep 2004.

[5] L. Schenato, B. Sinopoli, M. Franceschetti, K. Pooll, and S. Sastry, "Foundations of control and estimation over lossy networks," Proceedings of the IEEE, vol. 95(1), no. 1, pp. 163-187, Jan 2007.

[6] J. Silverstein and S. Choi, "Analysis of the limiting spectral distribution of large dimensional random matrices," Journal of Multivariate Analysis, vol. 54(2), pp. 295-309, 1995.

[7] R. Speicher, "Free convolution and the random sum of matrices," Pub. Res. Inst. Math. Sci., vol. 29, pp. 731-744, 1993.

[8] D. Voiculescu, "Asymptotically commuting finite rank unitary operators without commuting approximants," Acta Sci. Math., vol. 45, pp. 429-431, 1983.

[9] D. Voiculescu, "Addition of certain non-commuting random variables," Journal of Functional Analysis, vol. 66, pp. 1986, 1986.

[10] V. A. Marcenko and L. A. Pastur, "Distributions of eigenvalues for some sets of random matrices," Math. USSR-Sbornik, vol. 1, pp. 457483, 1967.

[11] A. M. Tulino and S. Verdu, "Random matrix theory and wireless communications," Foundations and Trends in communications and Information Theory, vol. 1, no. 1, pp. 1-182, 2004.

[12] R. Speicher, "Free probability theory and non-crossing partitions," in Seminaire Lotharingien de Combinatoire B39c, 1997.

[13] Z. D. Bai and J. W. Silverstein, "No eigenvalues outside the support of the limiting spectral distribution of large dimensional sample covariance matrices," Annals of Probability, vol. 26, pp. 316-345, 1998.

[14] B. Hassibi, A.H. Sayed, and T. Kailath, Indefinite Quadratic Estimation and Control: A Unified Approach to $H^{2}$ and $H^{\infty}$ Theories, SIAM, 1999. 